\title{
Moluscos de água doce da Floresta Nacional Mário Xavier, Seropédica, Rio de Janeiro
}

\author{
Isabela Cristina Brito Gonçalves ${ }^{1,2}$ (D) Igor Christo Miyahira $^{3}$ (D), Luiz Eduardo \\ Macedo de Lacerda ${ }^{1}$ (D), Renata de Freitas Ximenes ${ }^{1}$ (i) \& Sonia Barbosa dos \\ Santos $^{1,2}$ (D)
}

(1) Universidade do Estado do Rio de Janeiro, Instituto de Biologia Roberto Alcantara Gomes, Departamento de Zoologia, Laboratório de Malacologia Límnica e Terrestre, Rua São Francisco Xavier 524, Maracanã 20550-900, Rio de Janeiro, Brasil. E-mail: isabelabiouerj@yahoo.com.br

(2) Universidade do Estado do Rio de Janeiro, Instituto de Biologia Roberto Alcantara Gomes, Programa de Pós-Graduação em Ecologia e Evolução, Rua São Francisco Xavier 524, Maracanã 20550-900, Rio de Janeiro, Brasil. E-mail: malacosonia@gmail.com

(3) Universidade Federal do Estado do Rio de Janeiro, Centro de Ciências Biológicas e da Saúde, Instituto de Biociências, Departamento de Zoologia e Programa de Pós-Graduação em Biodiversidade Neotropical, Avenida Pasteur 458, Urca 22290-240, Rio de Janeiro, Brasil. E-mail: igor.c.miyahira@unirio.br

Gonçalves I.C.B., Miyahira I.C., Lacerda L.E.M., Ximenes R.F. \& Santos S.B. (2021) Moluscos de água doce da Floresta Nacional Mário Xavier, Seropédica, Rio de Janeiro. Pesquisa e Ensino em Ciências Exatas e da Natureza, 5: e1696. http://dx.doi.org/10.29215/pecen.v5i0.1696

Editor acadêmico: Silvio F. B. Lima. Recebido: 28 março 2021. Aceito: 19 abril 2021. Publicado: 27 abril 2021.

Resumo: A Floresta Nacional Mário Xavier (FLONA MX) é uma Unidade de Conservação (UC) de uso sustentável localizada no município de Seropédica, região metropolitana do estado do Rio de Janeiro. Foi criada em 1986, e desde então serviu como pioneira na produção de mudas e sementes de espécies nativas e não nativas. Atualmente, o conhecimento da fauna está mais restrito a espécies de vertebrados, especialmente peixes e anfíbios. O objetivo do presente estudo é apresentar um levantamento de moluscos límnicos da FLONA MX. Seis pontos de amostragem foram investigados, por quatro coletores durante 30 minutos em cada local de amostragem. Um total de seis espécies foram reportadas: Stenophysa marmorata (Guilding, 1828), Gundlachia ticaga (Marcus \& Marcus, 1962), Drepanotrema lucidum (Pfeiffer, 1839), Biomphalaria straminea (Dunker, 1848), Pomacea sp. e Omalonyx matheroni (Pontiez \& Michaud, 1835). Este trabalho traz informações originais sobre a presença de moluscos límnicos da FLONA MX e reforça a importância dessa UC como local para a preservação da fauna, podendo subsidiar futuros estudos de biologia, ecologia e conservação.

Palavras chave: Conservação, biodiversidade, Unidade de Conservação, moluscos límnicos, Gastropoda.

Freshwater molluscs of Floresta Nacional Mário Xavier, Seropédica, Rio de Janeiro

Abstract: Floresta Nacional Mário Xavier (FLONA MX) is a protected area of sustainable use located at the municipality of Seropédica, metropolitan area of Rio de Janeiro state. This protected area was created in 1986, being since this time a pioneer in the production of seedlings and seeds of native and non-native species. Nowadays, the knowledge about the fauna focuses on vertebrates as fishes and amphibians. The main goal of this study was to present a list of the freshwater molluscs occurring at FLONA MX. The field survey was done on six collecting stations by four collectors (30 minutes/collector). Six molluscan species were found: Stenophysa marmorata (Guilding, 1828), Gundlachia ticaga (Marcus \& Marcus, 1962), Drepanotrema lucidum (Pfeiffer, 1839), Biomphalaria straminea (Dunker, 1848), Pomacea sp. and Omalonyx matheroni (Pontiez \& Michaud, 1835).This work brings original information on the presence of molluscs on the area of FLONA MX and reinforces the importance of this protected area as a site for the preservation of fauna, being able to support future studies of biology, ecology and conservation. 
Key words: Conservation, biodiversity, protected areas, freshwater molluscs, Gastropoda.

\section{Introdução}

A Floresta Nacional Mario Xavier (FLONA MX) foi criada pelo Decreto Federal n ${ }^{\circ}$ 93.369, de 08 de outubro de 1986, a época colocada sob administração do Instituto Brasileiro do Meio Ambiente e dos Recursos Naturais Renováveis (IBAMA), e atualmente sob responsabilidade do Instituto Chico Mendes de Conservação da Biodiversidade (ICMBio). Corresponde à área do antigo Horto Florestal de Santa Cruz, criado em 1945 por Getúlio Vargas, em terras da antiga Fazenda Santa Cruz (Souza \& Vargas 2020). Segundo o Art. 17 da Lei ${ }^{\circ}$ 9.985, de 18 de julho de 2000, que institui o Sistema Nacional de Unidades de Conservação (SNUC), a Floresta Nacional é uma Unidade de Conservação de Uso Sustentável com cobertura florestal de espécies predominantemente nativas e tem por objetivo o uso múltiplo e sustentável dos recursos florestais e a pesquisa científica, com ênfase em métodos para exploração sustentável de florestas nativas (Brasil 2000). Apesar de estar inserida nessa categoria, a FLONA MX possui um grande percentual de área reflorestada com espécies introduzidas, como por exemplo, Eucalyptus sp. (Oliveira \& Bloomfield 1999). Alves \& Vargas (2019) a descrevem com uma cobertura de $16 \%$ de espécies nativas secundárias e o percentual restante de áreas de reflorestamento com inserção de espécies nativas de Mata Atlântica, em talhões exclusivos ou mistos com outras espécies não-nativas. Entre as principais utilizações sustentáveis da FLONA MX estão a produção de mudas e sementes, comercialização de toras, mourões e escoramentos de eucalipto, além de apoiar atividades de Educação Ambiental (INEA 2015; Souza \& Vargas 2020).

Durante as obras para a construção do Arco Metropolitano do Rio de Janeiro (Rodovia Raphael de Almeida Magalhães), inaugurado em julho de 2014, foi sugerido o aterramento de uma região alagada dentro da FLONA MX, onde foi reportada a presença de uma rã endêmica do Rio de Janeiro, Physalaemus soaresi Izechsohn, 1965 (Pontes et al. 2010; Souza 2017) classificada como Criticamente em Perigo (CR) no Livro Vermelho da Fauna Brasileira Ameaçada de Extinção (ICMBio/MMA 2018a,b). Após intensa mobilização da sociedade civil, o trajeto da estrada foi modificado e um viaduto foi construído para preservação da espécie (Souza \& Vargas 2020). A construção do Arco Metropolitano do Rio de Janeiro não foi o primeiro empreendimento de infraestrutura a impactar a área da FLONA MX. Anteriormente, a construção da rodovia Presidente Dutra (BR-116) dividiu a FLONA MX em duas áreas, quando ainda se chamava Horto Florestal de Santa Cruz (Souza 2017). Outra espécie ameaçada de extinção que também ocorre nesta UC é o peixe-das-nuvens, Notholebias minimus (Myers, 1942), categorizada também em CR (ICMBio/MMA 2018a,c). Apesar dos claros impactos encontrados na FLONA MX, a UC ainda preserva importantes remanescentes florestais e recursos hídricos. Estudos faunísticos e florísticos já foram realizados na FLONA MX, visando espécies de anuros (Izecksohn \& Carvalho-e-Silva 2001; Caram et al. 2016), artrópodes (Fernandes et al. 2011) e briófitas (Oliveira \& Wienskoski 2019).

Os moluscos de água doce constituem um importante grupo faunístico destes ambientes (Fransozo \& Negreiro-Fransozo 2016; Vaughn 2018). Rosenberg (2014) estimou mais de 5000 espécies de moluscos de água doce para o mundo, um número provavelmente subestimado. No Brasil, as estimativas são ainda mais imprecisas, tendo Simone (2006) apresentado 373 espécies nativas. A falta de conhecimento básico sobre as espécies com ocorrência no Brasil tem impedido apropriadas avaliações do status de conservação dos moluscos (Miyahira et al. 2017a; Santos \& Miyahira 2018). Entre as informações mais básicas que podem ser obtidas sobre as espécies, e que contribuem para essas avaliações, estão aquelas relacionadas a distribuição, uma vez que o dimensionamento de áreas de ocorrência é um dos critérios utilizados para a definição de estados de ameaça (IUCN 2019). 
Diante do exposto, o presente estudo teve por finalidade investigar a fauna de moluscos de água doce da FLONA MX, a fim de contribuir para o conhecimento da diversidade e subsidiar futuras estratégias de conservação.

\section{Material e Métodos}

A FLONA MX está localizada no município de Seropédica, na Região Metropolitana do Rio de Janeiro (Figura 1), sendo a única UC inserida na categoria de Floresta Nacional no estado do Rio de Janeiro (INEA 2015) delimitada por importantes rotas rodoviárias do estado do Rio de Janeiro como a BR-101, BR-465 e o Arco Metropolitano. No entorno da FLONA MX existem diversos loteamentos que exercem pressão sobre a UC, sendo desenvolvidas atividades de Educação Ambiental com a população local a fim de reconhecer sua importância e preservação (INEA 2015). A FLONA MX ocupa uma área estimada de 495,99 hectares, e está inserida no bioma Mata Atlântica, podendo ser considerada como um dos últimos fragmentos de Floresta Ombrófila Densa das Terras Baixas da bacia do rio Guandu (Lima \& Santos 1998; INEA 2015). Este rio é o principal da Região Hidrográfica II (Guandu), além de ser o manancial responsável pelo abastecimento de grande parte da Região Metropolitana do Rio de Janeiro (INEA 2011).

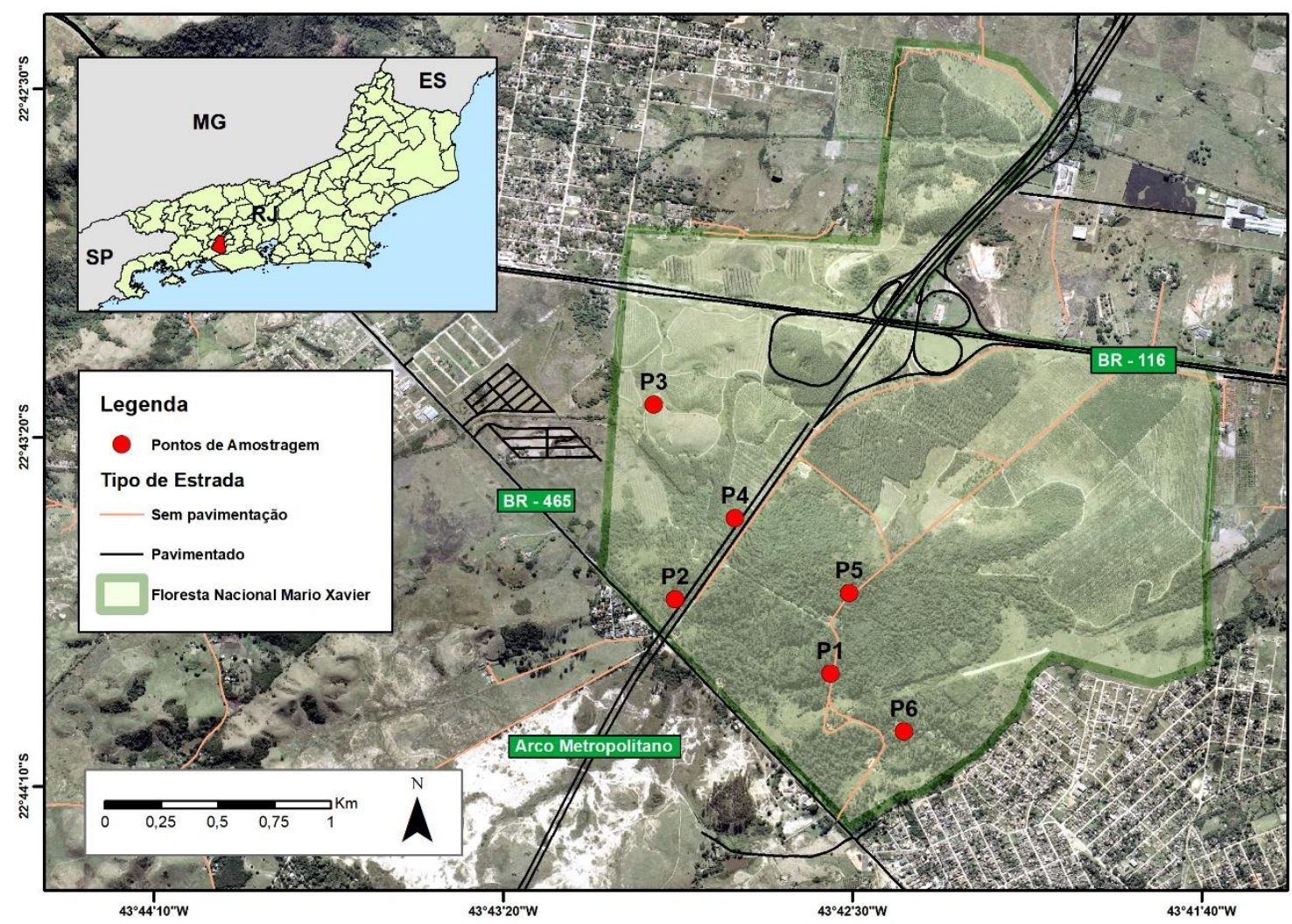

Figura 1. Pontos de amostragem na Floresta Nacional Mário Xavier (FLONA MX), Seropédica, RJ. São destacadas na imagem as rodovias que entrecortam a Unidade de Conservação; também é possível observar as pressões urbanas no entorno. No canto superior esquerdo, o estado do Rio de Janeiro, com o município de Seropédica em destaque.

O estudo foi realizado em outubro de 2010, com investigação em seis pontos de coleta abrangendo riachos e alagados desta UC: Ponto 1 - Poço do rio Drago; Ponto 2 - Lagoa próximo ao Arco Metropolitano do Rio de Janeiro; Ponto 3 - Brejo, próximo à Rodovia Presidente Dutra (BR-116); Ponto 4 - Brejo, próximo ao Centro de Triagem de Animais Silvestres (CETAS IBAMA); Ponto 5 - rio Drago; Ponto 6 - Valão das Louças, próximo a entrada da FLONA MX (Figuras 1-2). 
Quatro coletores munidos de concha de captura de moluscos e peneiras investigaram cada ponto de amostragem por 30 minutos, perfazendo um total de 120 minutos de esforço amostral em cada ponto. Os moluscos límnicos foram procurados em todos os ambientes favoráveis, como folhas, troncos, ou mesmo no substrato do fundo do corpo hídrico (Figura 2). Os animais foram transportados para o laboratório em frascos plásticos, anestesiados com mentol e fixados em álcool $70^{\circ}$. As conchas foram preservadas a seco. A identificação dos moluscos foi feita com base na literatura disponível (e.g., Santos 2003; Lacerda et al. 2015; Miyahira et al. 2017a; Cuezzo et al. 2020) e com auxílio de especialistas quando necessário. $\mathrm{O}$ material encontra-se depositado na Coleção de Moluscos da Universidade do Estado do Rio de Janeiro (UERJ).
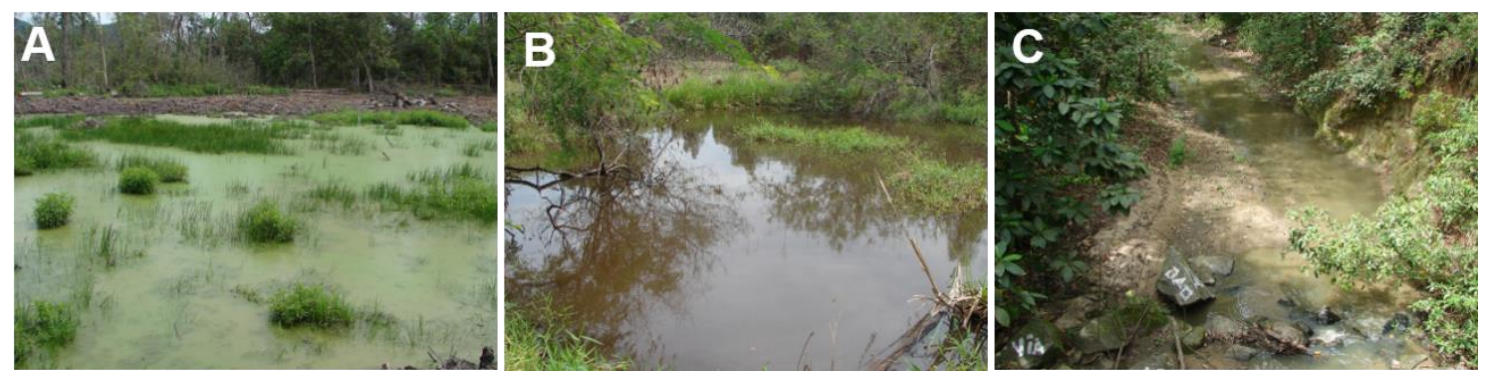

Figura 2. Diferentes habitats amostrados na Floresta Nacional Mário Xavier (FLONA MX): A. Pequena lagoa (Ponto 2); B. Brejo (Ponto 3); e C. Rio Drago (Ponto 5).

O mapa foi elaborado usando informação e imagem georreferenciada da Base Cartográfica do Estado do Rio de Janeiro (1:25.000) elaborada pelo Instituto Brasileiro de Geografia e Estatística (IBGE) e disponível em https://geoftp.ibge.gov.br/cartas_e_mapas/bases_ca rtograficas_continuas/bc25/rj/. As informações geográficas sobre a FLONA MX são derivadas de INEA (2011) e disponíveis em: http://www.inea.rj.gov.br/Portal/MegaDropDown/EstudosePublicac oes/EstadodoAmbiente/index.htm\&lang.

\section{Resultados e Discussão}

Foram reconhecidas para a FLONA MX seis espécies de moluscos distribuídas em quatro famílias: Ampullariidae, Pomacea sp.; Physidae, Stenophysa marmorata (Guilding, 1828); Planorbidae, Drepanotrema lucidum (Pfeiffer, 1839), Biomphalaria straminea (Dunker, 1848), Gundlachia ticaga (Marcus \& Marcus, 1962); e Succineidae, Omalonyx matheroni (Pontiez \& Michaud, 1835), uma espécie anfíbia (Figura 3, Tabela 1).

A presença de moluscos foi detectada em todos os seis pontos estudados. O ponto 2 (Figura 2A) apresentou a maior riqueza, com a ocorrência das seis espécies (Tabela 1). Ressaltase que o brejo deste ponto de amostragem foi um dos ambientes mais protegidos com a mudança de percurso e estrutura do Arco Metropolitano na área da FLONA MX. Apesar desta modificação ter sido pela ocorrência de Physalaemus soaresi, contribuiu para a preservação (ou resguardo) dessa considerável fauna de moluscos de água doce. No ponto 3 (Figura 2B) foi registrada a presença de três espécies. Nos pontos 4 e 6 ocorreram duas espécies, e nos pontos 1 e 5 (Figura 2C) apenas uma espécie foi reportada em cada. Não foi possível estabelecer de forma clara, uma relação entre o grau de preservação e a fauna encontrada. No município de Seropédica existem diferentes graus de esgotamento sanitário, no qual o rio Drago é um dos principais escoadores do esgoto do município (AGEVAP 2017). Somado a isso, no rio Drago (Figura 2C) podem ser observadas alterações ambientais como assoreamento, vegetação alterada nas margens, assim como outras ações antrópicas. Estes fatores podem ter levado aos menores valores observados nos pontos 1 e 5, ambos relacionados ao rio Drago. Gundlachia ticaga ocorreu em quatro pontos, apresentando a maior distribuição na área da FLONA MX (Tabela 1). Isto se deve provavelmente a capacidade desta espécie de se adaptar a grandes amplitudes de variação de matéria orgânica in natura, conforme apontado por Lacerda et al. (2011). Em geral, 
as abundâncias das espécies foram baixas, tendo Pomacea sp. o maior valor, seguido por Drepanotrema lucidum.

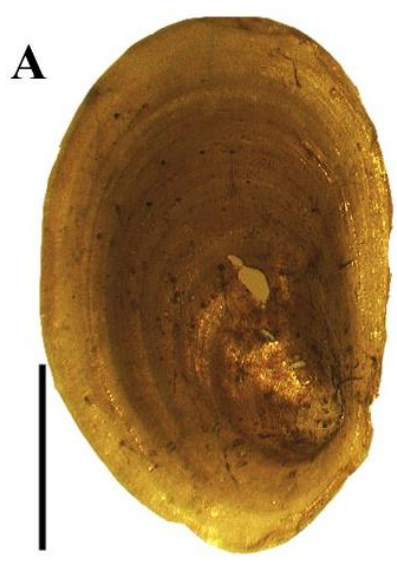

D

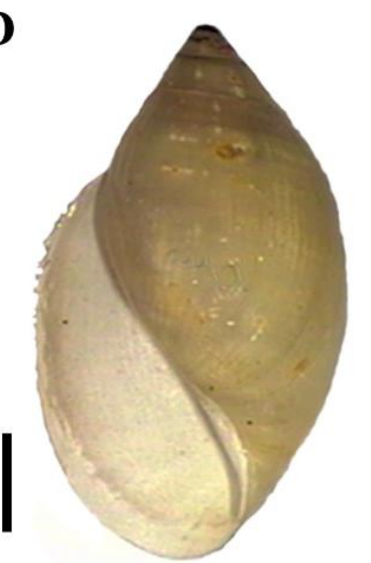

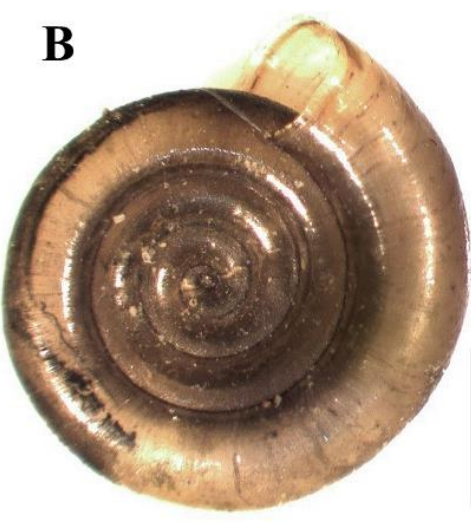

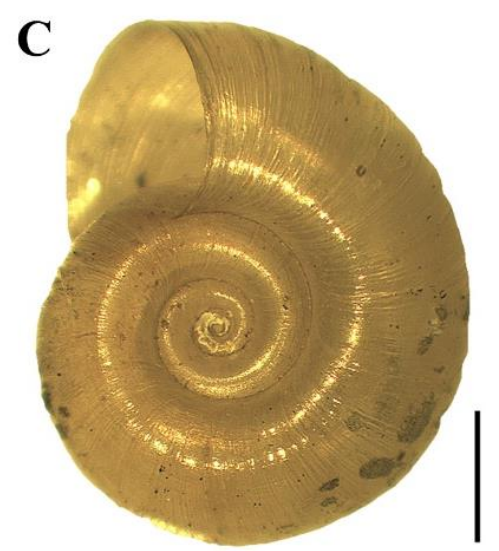

$\mathbf{E}$

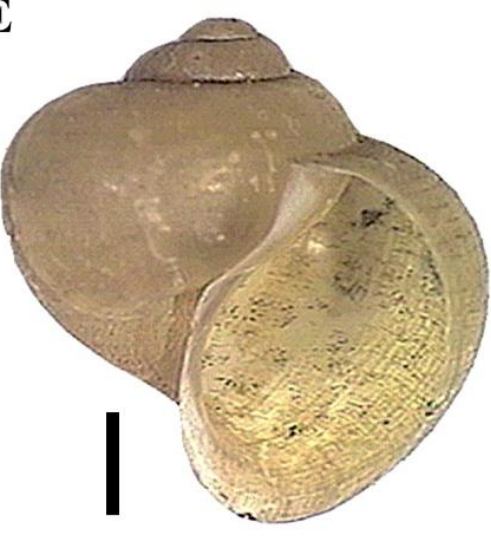

Figura 3. Espécies de moluscos de água doce encontrados na Floresta Nacional Mario Xavier, Seropédica, Rio de Janeiro: A. Gundlachia ticaga; B. Drepanotrema lucidum; C. Biomphalaria straminea; D. Stenophysa marmorata; e E. Pomacea sp. Escalas $=1 \mathrm{~mm}$.

Tabela 1. Abundância e número total de moluscos de água doce amostrados na Floresta Nacional Mario Xavier, com os números de depósito na Coleção de Moluscos da Universidade do Estado do Rio de Janeiro (UERJ).

\begin{tabular}{lccccccc}
\hline \multicolumn{1}{c}{ Espécies } & \multicolumn{9}{c}{ Pontos de Amostragem } & Material estudado \\
\hline & P1 & P2 & P3 & P4 & P5 & P6 & UERJ \\
\hline Pomacea sp. & - & 3 & 18 & - & - & 2 & $8152,8155,8161,8164$ \\
Stenophysa marmorata & - & 9 & 6 & - & 2 & - & $8153,8156,8160,8166$ \\
Drepanotrema lucidum & - & 17 & 1 & - & - & - & 8150,8157 \\
Biomphalaria straminea & - & 9 & - & 1 & - & - & $8151,8158,8162$ \\
Gundlachia ticaga & 2 & 2 & - & 1 & - & 3 & $8148,8154,8159,8162,8688$ \\
Omalonyx matheroni & - & 3 & - & - & - & - & 8149,8168 \\
\hline Total & 2 & 43 & 25 & 2 & 2 & 5 & \\
\hline
\end{tabular}

Na Tabela 2 são sumarizados os resultados do presente estudo, estando estes comparados com trabalhos prévios de moluscos de água doce em outras áreas do município de Seropédica. Thiengo et al. (2001) reportaram sete espécies de moluscos de água doce, enquanto que Miyahira et al. (2017b) relataram oito espécies para o município, sendo que cinco delas não foram mencionadas por Thiengo et al. (2001). A riqueza de seis espécies de moluscos encontradas na FLONA MX é similar as previamente reportadas (Thiengo et al. 2001; Miyahira et al. 2017b), embora a composição de espécies tenha sido diferente nos três estudos. As 
metodologias de coleta entre os três estudos são similares (utilização de concha de captura moluscos com busca ativa), contudo apresentam variações no número de coletores e tempo total de amostragem. Estes fatores podem ser a causa das diferenças observadas entre os estudos. Porém, como os três estudos retornaram riquezas similares, em áreas possivelmente equivalentes, podemos considerar as metodologias, e consequentemente os estudos, comparáveis. No presente estudo, três espécies foram registramos pela primeira vez para a região de Seropédica: Drepanotrema lucidum, Biomphalaria straminea e Omalonyx matheroni (Figura 3B). Considerando os três trabalhos (Thiengo et al. 2001; Miyahira et al. 2017b; presente estudo) foram registradas 17 espécies de moluscos límnicos em Seropédica, e a FLONA MX resguarda 35,3\% dessa diversidade. Cabe ressaltar, que provavelmente Pomacea sp. se refere a uma das espécies previamente encontradas na região, i.e. Pomacea maculata Perry, 1810, Pomacea canaliculata (Lamarck, 1822) ou Pomacea sordida (Swainson, 1823) (Tabela 1; Thiengo et al. 2001; Miyahira et al. 2017b); contudo, foram coletados apenas conchas e animais jovens que não permitiram a identificação na categoria específica.

Tabela 2. Tabela de moluscos de água doce (Gastropoda e Bivalvia) registradas para o município de Seropédica (Rio de Janeiro) com registros da literatura e do presente estudo, ressaltando que os trabalhos abordam diferentes áreas do município.

\begin{tabular}{|c|c|c|c|c|}
\hline Famílias & Espécies & Thiengo et al. (2001) & Miyahira et al. (2017b) & Presente estudo \\
\hline \multirow[t]{4}{*}{ Ampulariidae } & Pomacea maculata Perry, 1810 & - & $\mathrm{X}$ & - \\
\hline & Pomacea canaliculata (Lamarck, 1822) & $\mathrm{X}$ & - & - \\
\hline & Pomacea sordida (Swainson, 1823) & $\mathrm{X}$ & - & - \\
\hline & Pomacea sp. & - & - & $\mathrm{X}$ \\
\hline Thiaridae & Melanoides tuberculata (Müller, 1774) & - & $\mathrm{X}$ & - \\
\hline \multirow[t]{2}{*}{ Physidae } & Stenophysa marmorata (Guilding, 1828) & $\mathrm{X}$ & - & $\mathrm{X}$ \\
\hline & Physa acuta Draparnaud, 1805 & $\mathrm{X}$ & $\mathrm{X}$ & - \\
\hline Lymnaeidae & Pseudosuccinea columella (Say, 1817) & $\mathrm{X}$ & - & - \\
\hline \multirow[t]{6}{*}{ Planorbidae } & Drepanotrema lucidum (Pfeiffer, 1839) & - & - & $\mathrm{X}$ \\
\hline & Biomphalaria straminea (Dunker, 1848) & - & - & $\mathrm{x}$ \\
\hline & Biomphalaria tenagophila (d’Orbigny, 1835) & $\mathrm{X}$ & $\mathrm{X}$ & - \\
\hline & Gundlachia ticaga (Marcus \& Marcus, 1962) & $\mathrm{X}$ & - & $\mathrm{X}$ \\
\hline & Gundlachia radiata (Guilding, 1828) & - & $\mathrm{X}$ & - \\
\hline & Ferrissia californica (Rowell, 1863) & - & $\mathrm{X}$ & - \\
\hline Succineidae & Omalonyx matheroni (Pontiez \& Michaud, 1835) & - & - & $\mathrm{X}$ \\
\hline Hyriidae & Diplodon ellipticus Spix in Wagner, 1827 & - & $\mathrm{X}$ & - \\
\hline \multirow[t]{2}{*}{ Cyrenidae } & Corbicula fluminea (Müller, 1774) & - & $\mathrm{X}$ & - \\
\hline & Total & 7 & 8 & 6 \\
\hline
\end{tabular}

Biomphalaria straminea, registrada no presente estudo, e Biomphalaria tenagophila (d'Orbigny, 1835) referida também para o município por Thiengo et al. (2001) e Miyahira et al. (2017b), são vetores da esquistossomose, e representam um potencial risco de transmissão dessa doença. Thiengo et al. (2001) retrataram em seu trabalho alguns casos de esquistossomose para a região de Seropédica e Itaguaí. Embora nenhum caso tenha sido reportado para Seropédica entre 2010 a 2012 (Martinazzo \& Baroni 2014), a vigilância epidemiológica é necessária.

Duas das espécies encontradas na FLONA MX foram avaliadas na última revisão do Livro Vermelho da Fauna Brasileira Ameaçada de Extinção do Brasil (ICMBio/MMA 2018a,d). Stenophysa marmorata foi incluída na categoria Vulnerável (VU) com base em observação da redução de suas populações frente a introdução de espécies invasoras, como Physa acuta Draparnaud, 1805; sendo assim considerada uma espécie ameaçada. A outra espécie avaliada na última edição do Livro Vermelho foi Gundlachia ticaga, categorizada como Menos Preocupante 
(LC) com base em sua ampla distribuição, ou seja, sem riscos iminentes. As demais espécies nativas que ocorrem na FLONA MX não foram avaliadas, mas esses dados se tornam relevantes para uma próxima avaliação.

Miyahira et al. (2017b) identificaram para Seropédica duas espécies não-nativas, Melanoides tuberculata (Müller, 1774) e Ferrissia fragilis (Tryon, 1863) que não foram observadas dentro da FLONA MX. A primeira é uma espécie invasora com ampla distribuição no estado do Rio de Janeiro e no Brasil (Coelho et al. 2018; Miyahira et al. 2020). Pode provocar alterações nos sistemas onde é introduzida, incluindo deslocamento de espécies nativas (Freitas et al. 1987; Giovanelli et al. 2005; Santos et al. 2012; Braga et al. 2014). Ferrissia fragilis foi inicialmente identificada no Brasil em áreas dos estados do Rio de Janeiro e Minas Gerais (Lacerda et al. 2015; Miyahira et al. 2020). Miyahira et al. (2017b) ampliaram a distribuição desta espécie no estado do Rio de Janeiro, tendo localizado a mesma na bacia do rio Guandu. Provavelmente, deve estar mais amplamente distribuída do que os registros atuais apontam, pois o pequeno tamanho (3 a $5 \mathrm{~mm}$ ) dificulta a identificação, impedindo elucidar sua real distribuição (Lacerda et al. 2015). Atualmente, foi considerada sinonímia de Ferrissia californica (Rowell, 1863) por Christensen (2016). Efeitos negativos dessa espécie sobre a fauna nativa ainda são desconhecidos. Devem ser empregados esforços para evitar a dispersão destas espécies, especialmente $M$. tuberculata, para a FLONA MX, tendo em vista que ocorrem em áreas próximas. Melanoides tuberculata tende a dominar os ambientes nos quais é introduzida (Miyahira et al. 2010) e pode afetar negativamente não só as espécies nativas de moluscos de água doce, mas a partir de alteração da qualidade ambiental do habitat, as demais espécies de anuros e peixes, incluindo as ameaçadas.

\section{Considerações finais}

Apesar dos impactos ocorridos ao longo do tempo na FLONA MX, ela ainda sustenta um número de espécies de moluscos de água doce comparável à de outras áreas da região, constituindo uma importante área de conservação, onde temos a ocorrência de uma espécie ameaçada de moluscos de água doce, Stenophysa marmorata. Futuros estudos são necessários para subsidiar estratégias de conservação, incluindo acompanhamento das espécies encontradas e estudos de dinâmica populacionais e reprodutivos. Ações de Educação Ambiental com o objetivo de sensibilizar a população do entorno sobre a diversidade da fauna límnica abrigada pela FLONA MX, e suas conexões com os demais elementos faunísticos e florísticos, contribuirão para uma melhor percepção da importância da conservação dessa UC.

\section{Agradecimentos}

A equipe gestora da FLONA MX pela gentileza e apoio logístico; ICMBio (Instituto Chico Mendes de Conservação da Biodiversidade) pela licença 10812-1 para SBS; Dra Janine Oliveira Arruda (Museu de Ciências Naturais da Fundação Zoobotânica do Rio Grande do Sul) pela identificação de $O$. matheroni; Programa de Apoio à Pesquisa e Docência da UERJ (PAPDDocência, E-26/007/10662/2019) e a CAPES (bolsa de mestrado, período 2009-2011) pelas bolsas concedidas a LEML; Programa Prociência da UERJ pela bolsa de produtividade concedida à SBS; FAPERJ pela bolsa de mestrado concedida a ICM (E-26/100.815/2008); UERJ pela bolsa PIBICCNPq (período 2009-2010) concedida à ICBG; e aos revisores pelas sugestões e comentários.

\section{Referências}

AGEVAP (2017) Plano estratégico de recursos hídricos das bacias hidrográficas dos rios Guandu, da Guarda e Guandu Mirim. Diagnóstico - Tomo I. Rio de Janeiro, RJ. Disponível em: https://www.comiteguandu.org.br/plano-de-bacia.php (Acessado em 21/04/2021).

Alves A.G. \& Vargas K.B. (2019) Caracterização fitofisionômica dos principais talhões de espécies arbóreas da Floresta Nacional Mário Xavier-Seropédica-RJ. XVIII Simpósio Brasileiro de Geografia Física Aplicada 1 (1): 1-12. 
Braga R.M.R.B., Miyahira I.C., Lacerda L.E.M., Gonçalves I.C.B. \& Santos S.B. (2014) The influence of an invasive gastropod on the population dynamics of Pisidium punctiferum (Sphaeriidae) (p. 97-114). In: Piza A.R.T., Tallarico L.F., Introíni G.O. \& Santos S.B. (Eds). Medical and Applied Malacology Crossing Boundaries: Integrative Approaches to Malacology. Newcastle: Cambridge Scholars Publishing. 230 p.

Brasil. Ministério do Meio Ambiente. (2000) Regulamenta o art. 225, par. $1^{\text {o }}$, incisos i, ii, iii e vii da constituição federal, institui o sistema nacional de unidades de conservação da natureza e dá outras providências. Ministério do Meio Ambiente. Brasília: MMA.

Caram J., Gomes M.R., Luna-Dias C. \& Carvalho-e-Silva S.P. (2016) Updated list of anurans from Floresta Nacional Mário Xavier, Seropédica, Rio de Janeiro, Brazil: changes from 1990 to 2012. Check List, 12 (6): 1997. http://dx.doi.org/10.15560/12.6.1997

Christensen C.C. (2016) Change of status and name for a Hawaiian freshwater Limpet: Ancylus sharpi Sykes, 1900, is the invasive North American Ferrissia californica (Rowell, 1863), formerly known as Ferrissia fragilis (Tryon, 1863) (Gastropoda: Planorbidae: Ancylinae). Bishop Museum Occasional Papers, 118: 5-8.

Coelho P.N., Fernandez M.A., Cesar D.A.S., Ruocco A.M.C. \& Henry R. (2018) Updated distribution and range expansion of the gastropod invader Melanoides tuberculata (Müller, 1774) in Brazilian waters. BioInvasions Records, 7(4): 405-409. https://doi.org/10.3391/bir.2018.7.4.08

Cuezzo M.G., Gutiérrez Gregoric D.E., Pointier J.P, Vázquez A.A., Ituarte C., Mansur M.C.D., Arruda J.O., Barker G.M., Santos S.B., Ovando X.M.C., Lacerda L.E.M., Fernandez M.A., Thiengo S.C., Mattos A.C., Silva E.F., Berning I.B., Collado G.A., Miyahira I.C., Antoniazzi T.N., Pimpão D.M. \& Damborenea C. (2020) Phylum Mollusca. Capítulo 11 (p. 263-429). In: Damborenea C., Rogers C.D. \& Thorp J.H. (Eds). Keys to Neotropical and Antarctic Fauna. Volume 5. San Diego: Academic Press. 928 p.

Fernandes M.M., Magalhães L.M.S., Pereira M.G., Correia M.E.F., de Brito R.J. \& de Moura M.R. (2011) Influência de diferentes coberturas florestais na fauna do solo na Flona Mário Xavier, no município de Seropédica, RJ. Floresta, 41(3): 533-540. http://dx.doi.org/10.5380/rf.v41i3.24045

Fransozo A. \& Negreiros-Fransozo M.L. (2016) Zoologia dos Invertebrados. Rio de Janeiro: Roca. $661 \mathrm{p}$.

Freitas J.R., Bedê L.C., Marco Júnior P.D., Rocha L.A. \& Santos M.B.L. (1987) Population dynamics of aquatic snails in Pampulha reservoir. Memórias do Instituto Oswaldo Cruz, 82: 299-305. https://doi.org/10.1590/S0074-02761987000800057

Giovanelli A., Silva C.L.P.A.C.D., Leal G.B.E. \& Baptista D.F. (2005) Habitat preference of freshwater snails in relation to environmental factors and the presence of the competitor snail Melanoides tuberculatus (Müller, 1774). Memórias do Instituto Oswaldo Cruz, 100(2): 169-176.

ICMBio/MMA (2018a) Livro Vermelho da Fauna Brasileira Ameaçada de Extinção. Volume 1. $1^{\mathrm{a}}$ edição. Brasília, DF: Instituto Chico Mendes de Conservação da Biodiversidade / Ministério do Meio Ambiente. 492 p.

ICMBio/MMA (2018b) Livro Vermelho da Fauna Brasileira Ameaçada de Extinção. Volume V Anfíbios. $1^{\mathrm{a}}$ edição. Brasília, DF: Instituto Chico Mendes de Conservação da Biodiversidade / Ministério do Meio Ambiente. 131 p.

ICMBio/MMA (2018c) Livro Vermelho da Fauna Brasileira Ameaçada de Extinção. Volume VI Peixes. $1^{\mathrm{a}}$ edição. Brasília, DF: Instituto Chico Mendes de Conservação da Biodiversidade / Ministério do Meio Ambiente. 1235 p.

ICMBio/MMA (2018d) Livro Vermelho da Fauna Brasileira Ameaçada de Extinção. Volume VII Invertebrados. $1^{\mathrm{a}}$ edição. Brasília, DF: Instituto Chico Mendes de Conservação da Biodiversidade / Ministério do Meio Ambiente. 730 p.

INEA (2011) O estado do ambiente: indicadores ambientais do Rio de Janeiro. Rio de Janeiro: Secretaria Estadual do Ambiente; Instituto Estadual do Ambiente. 160 p. 
INEA (2015) Atlas das unidades de conservação do Estado do Rio de Janeiro. $2^{\circ}$ edição. São Paulo: Metalivros. $171 \mathrm{p}$.

IUCN Standards and Petitions Committee (2019) Guidelines for Using the IUCN Red List Categories and Criteria. Version 14. Prepared by the Standards and Petitions Committee.

Izecksohn E. \& Carvalho-e-Silva S.P. (2001) Anfíbios da Floresta Nacional Mário Xavier, município de Seropédica, estado do Rio de Janeiro, Brasil (Amphibia: Anura). Contribuições Avulsas Sobre a História Natural do Brasil, 39: 1-3.

Lacerda L.E.M., Miyahira I.C. \& Santos S.B. (2011) Shell morphology of the freshwater snail Gundlachia ticaga (Gastropoda, Ancylidae) from four sites in Ilha Grande, southeastern Brazil. Zoologia, 28: 334-342. http://dx.doi.org/10.1590/S1984- 46702011000300007

Lacerda L.E.M., Richau C.S., Amaral C.R.L., Silva D.A., Carvalho E.F. \& Santos S.B. (2015) Ferrissia fragilis (Tryon, 1863): a freshwater snail cryptic invader in Brazil revealed by morphological and molecular data. Aquatic Invasions, 10(2): 157-168. http://dx.doi.org/10.3391/ai.2015.10.2.04

Lima J.P.C. \& Santos L.A.F. (1998) Floresta Nacional Mário Xavier uma proposta de planejamento e de gestão ambiental-justificativa. Floresta e Ambiente, 5: 216-218.

Martinazo A.L. \& Baroni M.N. (2014) Plano Municipal de Saúde de Seropédica/RJ - 2014-2017. Prefeitura de Seropédica. 158 p.

Miyahira I.C., Lacerda L.E. \& Santos S.B. (2010) How many species are introduced every day? Some insights from a tropical insular stream in Brasil. Tentacle, 18: 30-32.

Miyahira I.C., Santos S.B. \& Mansur M.C.D. (2017a) Freshwater mussels from South America: state of the art of Unionida, specially Rhipidodontini. Biota Neotropica, 17(4): e20170341. https://doi.org/10.1590/1676-0611-bn-2017-0341

Miyahira I.C., Carneiro J.B., Gonçalves I.C.B., Lacerda L.E.M., Oliveira J.L., Vasconcelos M.C. \& Santos S.B. (2017b) Freshwater mollusks and environmental assessment of Guandu River, Rio de Janeiro, Brazil. Biota Neotropica, 17(3): e20170342. http://dx.doi.org/10.1590/1676-0611-bn2017-0342

Miyahira I.C., Pereira L.S. \& Santos L.N. (2020) Non-native freshwater molluscs in the Neotropics: what can be learned from Brazilian reservoirs? Aquatic Invasions, 15(3): 455-472. http://dx.doi.org/10.3391/ai.2020.15.3.06

Oliveira R.T. \& Blommfield V.K. (1999) Trilha auto guiada: proposta de implantação e interpretação na Floresta Nacional Mário Xavier Sandra Regina da Costa. Floresta e Ambiente, 6(1): 138-143.

Oliveira R.G. \& Wiensoski M.B. (2019) Briófitas da Floresta Nacional Mário Xavier (FLONA MX), Seropédica - RJ, Brasil. V Simpósio de Pesquisa em Mata Atlântica: 49-50.

Pontes J., Lima V., Pontes R., Santa-Fé C. \& Rocha C. (2010) Amphibia, Anura, Leiuperidae, Physalaemus soaresi Izecksohn, 1965: New record, distribution extension and geographic distribution map. Check List, 6: 159. https://doi.org/10.15560/6.1.159

Rosenberg G. (2014) A new critical estimate of named species-level diversity of the recent Mollusca. American Malacological Bulletin, 32(2): 308-322. http://dx.doi.org/10.4003/006.032.0204

Santos S.B. (2003) Estado atual do conhecimento dos ancilídeos na América do Sul (Mollusca, Gastropoda, Basommatophora). Revista de Biología Tropical 51 (3): 191-223.

Santos S.B. \& Miyahira I.C. (2018) New evaluation of the list of endangered non-marine molluscs in Brasil in progress. Tentacle, 26: 10.

Santos S.B., Thiengo S.C., Fernandez M.A., Miyahira I.C., Gonçalves I.C.B., Ximenes R.F., Mansur M.C.D. \& Pereira D. (2012) Espécies de moluscos límnicos invasores no Brasil (p. 25-49). In: Mansur M.C.D., Santos C.P., Pereira D., Paz I.C.P., Zurita M.L.L., Rodriguez M.T.R., Nehrke M.V. \& Bergonci P.E.A. (Eds). Moluscos límnicos invasores no Brasil: biologia, prevenção e controle. Porto Alegre: Redes Editora. 412 p.

Simone L.R.L. (2006) Land and Freshwater Mollusks of Brazil. São Paulo: EGB, FAPESP. 390 p.

Souza R.L.N. (2017) Restauração da Mata Atlântica: potencialidades, fragilidades, e os conflitos ambientais na floresta nacional Mário Xavier, Seropédica/RJ. Dissertação (Programa de Pós- 
Graduação em Geografia). Universidade Federal Rural do Rio de Janeiro, Seropédica, Rio de Janeiro.

Souza T.R.R.S. \& Vargas K.B. (2020) Flona Mário Xavier: Entre Histórias e Memórias. Seropédica (E-Book): editora. $20 \mathrm{p}$.

Thiengo S.C., Fernandez M.A., Boaventura M.F., Grault C.E., Silva H.F., Mattos A.C. \& Santos S.B. (2001) Freshwater snails and schistosomiasis mansoni in the state of Rio de Janeiro, Brazil: IMetropolitan Mesoregion. Memórias do Instituto Oswaldo Cruz, 96: 177-184. https://doi.org/10.1590/S0074-02762001000900028

Vaughn C.C. (2018) Ecosystem services provided by freshwater mussels. Hydrobiologia, 810(1): 15-27. https://doi.org/10.1007/s10750-017-3139-x 